\title{
UNIO LITURGICA. DIE KULTISCHE EINSTIMMUNG IN GÖTTERWELTLICHEN LOBPREIS ALS GRUNDMOTIV "ESOTERISCHER" ÜBERLIEFERUNG IM ALTEN ÄGYPTEN.
}

\author{
Jan Assmann \\ Universität Heidelberg
}

Für Peter Schäfer

\section{Die Theorie der heiligen Sprache als Göttersprache nach Iamblich und den graeco-ägyptischen Zauberpapyri}

Dem Neuplatoniker Jamblichos verdanken wir eine Darstellung der "ägyptischen Mysterien" und darin auch eine Erläuterung der theurgischen Kraft des Gebets. ${ }^{1}$ In diesem Zusammenhang begründet er den Vorrang der alten Sprachen wie Babylonisch und Ägyptisch. Es sind heilige Sprachen: "Weil nämlich die Götter die gesamten Sprachen der heiligen Völker wie der Ägypter und Assyrer für heilig erklärt haben, sind wir der Ansicht, daß unser mündlicher Verkehr mit den Göttern sich in jener Ausdrucksweise abwickeln müsse, die den Göttern verwandt ist. Auch ist die Form der Aussprache mit den Göttern derart die ursprünglichste und älteste ... Deshalb also halten wir an dem Gesetze ihrer Überlieferung unerschütterlich fest, da diese Form den Göttern eignet und ihnen angepaßt ist." (VII.4) "Man muß also die altehrwürdigen Gebetsformeln wie heilige Asyle behüten, immer als die gleichen und in gleicher Weise, während man weder irgendetwas von ihnen wegnimmt, noch ihnen irgendetwas von anderswo zusetzt." (VII.5). Die Griechen, so fährt er fort, sind neuerungssüchtig, "haben nichts Festes in sich und bewahren nichts so, wie sie es von irgendwem erhalten haben... Die Barbaren dagegen bleiben stets standhaft bei denselben Formeln, da sie von konservativem Charakter sind; eben deshalb aber sind sie sowohl den Göttern lieb als auch bringen sie den Göttern Formeln dar, die ihnen angenehm

\footnotetext{
${ }^{1}$ Iamblichus, De mysteriis Aegyptionum, VII ed. E. des Places, Collection Budé, ${ }^{2} 1989$, 188-195; Übers. Th. Hopfner, Jamblichus, Ueber die Geheimlehren. Die Mysterien der Aegypter, Chaldäer und Assyrer, Schwarzenburg 1978, 162-168.
} 
sind. Diese Formeln aber zu verändern, ist keinem Menschen unter gar keinen Umständen erlaubt."

Der heilige Text ist wortlautgebunden, unübersetzbar und unveränderbar. Er "bezeichnet" nicht das Heilige mit Hilfe "konventioneller" Zeichen, sondern ist selbst heilig, d.h. dem Heiligen wesensverwandt. Er vermag es daher zu vergegenwärtigen, zu "präsentifizieren". ${ }^{2}$ Das ist der Sinn des theurgischen Gebets. Es kommt nicht darauf an, diesen Text zu verstehen. Nicht das Herz, der mitvollziehende Intellekt und das vom Heiligen ergriffene Gemüt werden hier gefordert, sondern allein die präzise Aussprache, die in allen Einzelheiten korrekte Aus- und Aufführung der Vorschrift. ${ }^{3}$

Die gleiche Anschauung über den mystischen Charakter der Heiligen Sprache ${ }^{4}$ vertritt auch das Corpus Hermeticum. Im Eingang zu Traktat XVI geht es um das Problem der Übersetzbarkeit heiliger Texte aus dem Ägyptischen ins Griechische. Die heiligen Texte sind nicht übersetzbar, weil sie ihre theurgische "Energie" nur in der Ursprache entfalten können. Diese Unübersetzbarkeit ist ein konstituierendes Element jener Geheimheit und Esoterik, mit der esoterische Traditionen umgeben sind.

Er sagte, daß die Leser meiner Bücher (Hermes Trismegistos spricht) glauben werden, daß sie klar und schlicht geschrieben seien, während sie doch ganz im Gegenteil unklar sind und die Bedeutung der Worte verhüllen und vollkommen dunkel sein werden, wenn eines Tages die Griechen sie aus unserer Sprache in die ihre übersetzen wollen, was zur vollständigen Verzerrung und Verdunkelung des Textes führen wird.

${ }^{2}$ J.P. Vernant, "De la presentification de l'invisible a l'imitation de l'apparence", in: Image et Signification, Rencontres de l'Ecole du Louvre (1983) 25 ff., $293 \mathrm{ff}$.

${ }^{3}$ Zur Vorstellung von der theurgischen Kraft der barbarikà onómata vgl. Cumont, Rel. Or. ${ }^{4}, 87,240$ n. 72; 295 n. 90; Eg.d.astrol., 125; Bidez-Cumont, Les mages hellénisés, II, 69 n. 14; Th. Hopfner, Griechisch-ägyptischer Offenbarungszauber, ${ }^{2} 1974-1990$, Bd. I, $\S \S$ 718-21, 724-25; Orac. Chald., p. 58 Kroll; Psellos, Expos. Orac. Chald. 1132 C Migne; Clemens Alexandrinus, Strom. I 21, 146; Origenes, c. Celsum I, 24-25, 28; V 45; Euseb., pr. evang. IV, 1,11. Vgl. auch M.P. Nilsson, Geschichte der griechischen Religion, 2.Bd., München ${ }^{4} 1988,448-454$.

"Interessanterweise verbindet sich diese "mystische" Vorstellung einer heiligen Sprache in der späteren Überlieferung nicht mit der Sprache, sondern mit der Schrift der Ägypter, vgl. z.B. Giordano Bruno, De magia (Op. lat. III, 411-12, nach Fr. Yates, Giordano Bruno and the Hermetic Tradition, 263) “... the sacred letters used among the Egyptians were called hieroglyphs ... which were images... taken from the things of nature, or their parts. By using such writings and voices (voces), the Egyptians used to capture with marvellous skill the language of the gods. Afterwards when letters of the kind which we use now with another kind of industry were invented by Theuth or some other, this brought about a great rift both in memory and in the divine and magical sciences." 
In der Originalsprache bringt der Text seine Bedeutung klar zum Ausdruck, denn die reine Lautqualität und die Intonation der ägyptischen Worte enthalten die Kraft der gemeinten Sache.

$\mathrm{La} ß$ diesen Text daher unübersetzt, damit diese Geheimnisse den Griechen entzogen bleiben und damit ihre freche, kraftlose und schwülstige Redeweise die Würde und Kraft unserer Sprache und die Energie der Namen nicht zum Verschwinden bringt. Denn die Griechen haben nur leere Reden, gut zum Imponieren, und ihre Philosophie ist bloß geschwätziger Lärm. Wir dagegen, wir gebrauchen nicht Wörter,

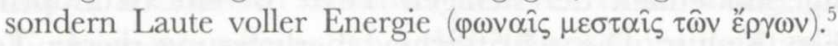

Die energetische Theorie der Sprache ist magisch. Die magische Kraft der Zaubersprüche liegt in ihrer Lautgestalt. Der Laut, die sinnliche Klangqualität der Sprache ist es, die die Macht hat, die göttliche Sphäre zu erreichen. Diese energetische Dimension der Sprache ist unübersetzbar. Man muß sie in der Originalgestalt reproduzieren.

Ein Blick in die zeitgenössische graeco-ägyptische Zauberliteratur lehrt, wovon Jamblichus spricht. In der "Leidener Weltschöpfung" 6 (um nur ein Beispiel unter zahllosen anderen zu nennen) ruft der

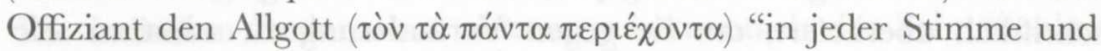
in jeder Sprache" an, womit aber nicht Menschen -, sondern Göttersprache gemeint ist: zuerst mit den Worten des Sonnengottes: AX EBY KPSM, sodann mit den Worten der "ersterschienenen Boten":

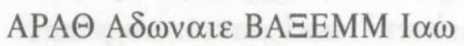

Der erste Bote ruft in der Vogelsprache: APAI, das heißt "Wehe meinem Feind"...

aber Helios besingt dich in der Hieroglyphensprache: $\Lambda$ AI $\Lambda$ AM und hebräisch mit demselben Namen. Dann spricht er

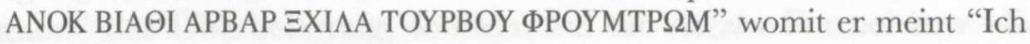
gehe dir voran,

Herr, ich der N.N., der deinetwegen auf der Barke aufgeht" usw. usw.

Hier wird nicht nur Ägyptisch und Assyrisch gesprochen. Vogelgestaltige Götter sprechen die Vogelsprache ("orneoglyphisch": ó $\rho \varepsilon \varepsilon \gamma \lambda v-$

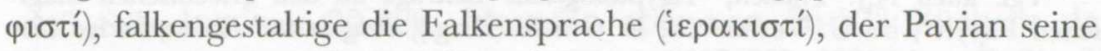

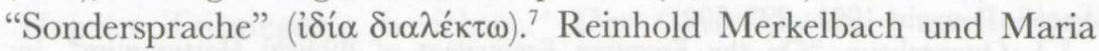

${ }^{5}$ Corpus Hermeticum XVI ed. A.J. Festugière, A.D. Nock II, 230; G. Fowden, The Egyptian Hermes. An Historical Approach to the Late Pagan Mind, Cambridge 1986, 37.

${ }^{6}$ R. Merkelbach, M. Totti, Abrasax. Ausgewählte Papyri religiösen und magischen Inhalts, Bd. 3: Zwei griechisch-ägyptische Weihezeremonien (Die Leidener Weltschöpfung. Die Pschai-Aion-Liturgie), Abh. der rhein.-westfäl. Ak.d. Wiss., Sonderreihe Papyrologica Coloniensia XVII.3, Opladen 1992.

7 Vgl. den Beitrag von H.D. Betz ("baboonic") mit Hinweis auf Hopfner, Offenbarungszauber $1, \S 780$ und W. Speyer, "Die Nachahmung von Tierstimmen durch 
Totti haben in ihrem Kommentar mit großem Scharfsinn diese Zauberworte entschlüsselt und - in dem meisten Fällen sehr überzeugendals Transkriptionen aus dem Ägyptischen rekonstruiert. ${ }^{8}$

\section{Heilige Texte, Götterrede und göttliche Verfasserschaft in der altägyptischen Überlieferung}

Die Wortlautgebundenheit der heiligen Texte ist ein Grundprinzip der ägyptischen Kultur. Die schriftliche Überlieferung dieser Texte erscheint unter den allerfrühesten Bereichen, in denen sich die Ägypter die Möglichkeiten schriftlicher Aufzeichnung zunutze gemacht haben. Schon in den bildlichen Darstellungen des Rituals aus dem Alten Reich treffen wir auf den Mann mit der Papyrusrolle, den Vorlesepriester, der dann in den griechischen Texten als "Hierogrammateus" bezeichnet wird. Sie tragen auch den Titel "Chef", äg. hrj-tp, wovon hebräisches hartummim kommt. ${ }^{9}$ Schrift und schriftliche Überlieferung bilden das Grundgerüst des ägyptischen Kults. Heilige Texte werden schriftlich überliefert, um sie gegen Veränderung zu schützen. Sie fungieren als Partituren, die im Kult zur Auffuihrung gebracht werden..$^{10}$

Die Texte, die man diese Vorlesepriester auf den Darstellungen rezitieren sieht, werden "Verklärungen" genannt, ägyptisch $s^{3} h w$, die Kausativ-Form des Stammes 3 h, der "leuchten" und davon abgeleitet "Geist sein" heißt." Allein schon die Kausativbildung dieser Gattungsbezeichnung zeigt, daß wir es hier mit wirkungsvollen Texten zu tun haben, deren Rezitation eine Transformation - nämlich in den $\mathrm{Zu}$ -

Besessene (Zu Platon, resp. 3, 396b)", in Ders., Frühes Christentum im antiken Strahlungsfeld (WUNT 50, Tübingen 1989), 193-198. Zur Sprache der Paviane vgl. besonders H. te Velde, "Some Remarks on the Mysterious Language of the Baboons", in: J.H. Kàmstra (Hrsg.), Funerary Symbols and Religion. Essays dedicated to Prof. Heerma van Voss, Kampen 1988, 129-136, der allerdings auf die magischen Papyri nicht eingeht.

${ }^{8}$ Vgl. auch H.J. Thissen, "Ägyptologische Beiträge zu den griechischen magischen Papyri”, in: U. Verhoeven, E. Graefe (Hrsg.), Religion und Philosophie im Alten Ägypten, Louvain 1991, 293-302.

"J. Quaegebeur, "On the Egyptian Equivalent of Biblical Hartummim", in: Pharaonic Egypt, the Bible and Christianity, Jerusalem 1985, 162-172; ders., "La designation (P')hry-tp: PHRITOB", in: Osing, J., Dreyer, G. (Hgg.), Form und Maß, Fs Fecht, Wiesbaden 1987, 368-394.

${ }_{10}$ Vgl. Manfred Weber, Beiträge zur Kenntnis des Schrift- und Buchwesens der alten Ägypter, Diss. Köln 1969; ders., "Lebenshaus", in: LÄ III, 1979, 954 ff. Allg. zur ägyptischen Schriftkultur: A. Schlott, Schrift und Schreiber im Alten Agypten, München 1989 (in dem hier interessierenden Punkt leider unergiebig).

11 Vgl. hierzu Verf., Ägypten - Theologie und Frömmigkeit einer frühen Hochkultur, Stuttgart 1984, 4.Kapitel; ders., Art. "Verklärung", in: LA VI, 998-1006. 
stand $3 h$ - bewirkt. Diese Texte, die uns in großer Zahl erhalten sind, enthalten zwar keine Zaubernamen. Sie sind auch in völlig normalem Ägyptisch abgefaßt. Aber die Schriftlichkeit ihrer Überlieferung und Aufführung verweist auf den Sonderstatus ihrer Sprachgestalt. Sie ist sakrosankt, und die Schrift dient als Mittel ihrer Bewahrung. Der Priester mit der Schriftrolle garantiert die Genauigkeit der Aufführung, genau wie im modernen Musikleben der Dirigent mit der Partitur. Dabei bedarf es keiner weiteren Erläuterung, daß die Schrift im Funktionszusammenhang der "Rezitationsliteratur" nur als ein Hilfsmittel fungiert, als ein Zwischenspeicher. Der eigentliche Kommunikationsakt ist die mündliche Aufführung. Die Schrift wird nicht "gelesen". Auch darin entspricht diese Art der rituellen Schriftlichkeit vollkommen der musikalischen Partitur. Auch diese will ja nicht gelesen, sondern aufgeführt werden. Selbstverständlich kann der Text anstatt schriftlich aufgezeichnet auch auswendig gelernt werden. Theoretisch macht das gar keinen Unterschied. Das menschliche Gedächtnis fungiert hier als Zwischenspeicher und "Notationssystem". Die indischen Brahmanen mißtrauen bekanntlich der Schriftlichkeit und ziehen das Gedächtnis als Zwischenspeicher vor. Die Ägypter haben von Anfang an umgekehrt optiert. Sie mißtrauten dem Gedächtnis und haben sich die Schrift für die Speicherung kultischer Texte zunutze gemacht.

Im Medium dieser geheiligten und heiligenden Schriftlichkeit entsteht aber keine "Heilige Schrift."12 Dazu haben es die Ägypter nie gebracht. Das von Jamblich zitierte Verbot der Veränderung, Hinzufügung oder Weglassung bezieht sich nicht auf das Insgesamt, den "Kanon" der Überlieferung, sondern auf die einzelne Formel bzw. die sakrosankte Sprachgestalt des einzelnen Spruchs, dessen theurgische oder "präsentifikatorische" Kraft auf eben dieser Sprachgestalt beruht. Geheiligt ist der Text, nicht der Textkanon. ${ }^{13}$ Dessen Grenzen verlieren sich vielmehr im Unabsehbaren. So schreibt Jamblich etwa, daß Seleukos von 20000 , Manetho aber von 36525 Büchern des Hermes Trismegistos berichtet. ${ }^{14}$ Der Gesamtbestand an heiligem Schrifttum ist uferlos und die Vorstellung eines Kanons ist dieser

12 Vgl. C. Colpe, "Heilige Schriften", in: Reallexikon für Antike und Christentum, Lieferung 106, 1987, 184-223.

${ }^{13}$ Zur Kanonformel vgl. Verf., Das kulturelle Gedächtnis. Schrift, Erinnerung und politische Identität in frühen Hochkulturen, München 1992, 103-106, $236 \mathrm{f}$.

${ }_{14}$ De Myst. VIII.1. 
Schriftkultur fremd. ${ }^{15}$ Worauf es vielmehr ankommt, ist der einzelne Text. Innerhalb dieses Stroms oder Ozeans heiliger Texte gibt es viele Gattungen und die Beschwörungsgebete, Verklärungen, Eulogien, Hymnen, Weihezeremonien und sonstigen Rezitationen von theurgischer, präsentifikatorischer Kraft bilden nur eine Untergruppe.

Von außen gesehen verlagert und erweitert sich dieser Strom ständig, und es kann überhaupt keine Rede davon sein, daß die "Barbaren" im Unterschied zu den Griechen keine Neuerungen zuließen und strikt am geheiligten Bestand uralter Texte festhielten. Natürlich kommen ständig neue Texte dazu, und insbesondere die graecoaegyptischen Zaubertexte tragen das unverkennbare Gepräge ihrer Zeit und reichern einen Grundbestand spätägyptischer Theologie mit allen möglichen jüdischen, iranischen, babylonischen und griechischen Versatzstücken an. Eine durchaus "moderne" Literatur entsteht hier. Von innen gesehen, d.h. im Selbstverständnis dieser Texte, wird hier aber eine uralte Überlieferung weitergegeben, und je moderner der Spruch, desto älter, heiliger, göttlicher, "authentischer" ist sein angeblicher Ursprung.

Im Berliner Museum gibt es einen Papyrus aus frühptolemäischer Zeit mit einer Sammlung von "Verklärungen", die sich in einem einleitenden Vermerk als Abschrift einer Handschrift aus der Zeit Amenophis' III. (ca. 1400-1360) ausgibt. ${ }^{16}$ Man würde das natürlich für den üblichen frommen Betrug halten. Ein Blick auf diese Texte lehrt jedoch, daß diese Sammlung noch wesentlich älter als die angegebene Vorlage ist und die einzelnen hier zusammengestellten Texte noch einmal sehr viel weiter zurückgehen. Dieselbe Textzusammenstellung findet sich bereits auf Särgen des frühen 2.Jahrtausends, und die einzelnen Texte stehen bereits in den Pyramiden der 6. Dynastie (24.Jh.v.Chr.). Die Textüberlieferung ist verblüffend gut. Hier ist also in der Tat eine Verklärungs- Liturgie im Rahmen der rituellen Schriftlichkeit über 2000 Jahre und mehr hinweg getreulich gespeichert und im Kult immer wieder wortlautgetreu zur Aufführung gebracht worden. Was um 2300 v.Chr. einmal "normales" Ägyptisch war, ist freilich um 300 v.Chr. längst eine Fremdsprache geworden, an der man

${ }^{15}$ Vgl. jedoch die "42 hochverbindlichen (pány anankaîa: = "Kanonischen"?) Büchern des Hermes", die nach Clemens Alexandrinus, Stromat. VI.4.35-7 von den ägyptischen Priestern in Prozession getragen wurden.

${ }_{16}$ G. Möller, Über die in einem späthieratischen Papyrus des Berliner Museums enthaltenen Pyramidentexte. Berlin 1900. 
im Kult festhielt aus Gründen, die Jamblich gewiß sehr treffend mit seinem den "Barbaren" zugeschriebenen Konservatismus diagnostiziert: die Götter lieben keine Neuerungen. Dieser Bruch zwischen normalem gesprochenen und geschriebenen Ägyptisch und dem in den Schriftrollen der Vorlesepriester bewahrten Sprachstadium läßt sich schwer datieren. Es handelt sich dabei zweifellos um einen kontinuierlichen Prozeß der Auseinanderentwicklung. Lange Zeit hat man den Unterschied zwischen Kultsprache und Alltagssprache gewiß nur als dialektale Variante empfunden. Spätestens nach der Amarnazeit wird den Ägyptern klar, daß sie es mit zwei verschiedenen Sprachen zu tun haben. Nun wird die alte Schriftsprache eigens in der Schule erlernt. ${ }^{17}$ In der Spätzeit ist die Kenntnis dieser Sprache zum Exklusivbesitz der Priester, und diese Sprache selbst zur exklusiven Kultsprache geworden, ebenso wie die Hieroglyphenschrift und deren Buchkursive, das Hieratische, in der sie geschrieben wird. In dieser Zeit dürfte sich die alte Bezeichnung für die Hieroglyphen, "Göttersprache" (mdt ntr) auch auf das darin aufgezeichnete, zur Fremdsprache gewordene Alt- und Mittelägyptisch ausgedehnt haben: Kultsprache ist Göttersprache. ${ }^{18}$

Die wirklich alte Liturgie des Pap. Berlin 3057 hat es nun offenbar nicht nötig, sich durch phantastische Fundlegenden oder gar göttliche Verfasserschaft zu legitimieren. Anders steht es mit der modernen Rezitationsliteratur der Spätzeit. An erster Stelle sind hier die verschiedenen "Briefe vom Atmen" ( $\left.\breve{s}^{c} t n s n s n\right)$ zu nennen, die in der Ptolemäerzeit neben dem althergebrachten Totenbuch entstehen. ${ }^{19}$ Sie werden der Göttin Isis als Verfasserin zugeschrieben.. Andere Bücher soll Thoth geschrieben haben. ${ }^{20}$

Die Fiktion göttlicher Verfasserschaft antwortet auf das Problem, die rapiden und grundlegenden Wandlungen religiöser Erkenntnis, Erfahrung und Sensibilität und das daraus erwachsende Bedürfnis nach religiösem Sinn in Einklang zu bringen mit der Überzeugung, daß nur das im Medium der rituellen Schriftlichkeit seit Urzeiten Gespeicherte einen Anspruch auf Wahrheit erheben darf. Wahrheit

17 Vgl. F. Junge, "Sprachstufen und Sprachgeschichte", in: Zeitschr.d.dt. Morgenländischen Ges., Suppl. VI, XXII. Deutscher Orientalistentag Stuttgart 1985, 17-34.

${ }_{18} \mathrm{Vgl}$. hierzu auch die treffenden Bemerkungen von H. te Velde, a.a.O., $134 \mathrm{f}$.

${ }^{19}$ J.C. Goyon, Rituels funéraires de l'ancienne Egypte, Paris 1972, 189ff.; ders., "La littérature funéraire tardive", in: Textes et langages de l'Égypte pharaonique III. Kairo 1974, 73-81.

${ }^{20}$ Schott, ZÄS 99, 1972, 20-25. Boylan, Thoth, the Hermes of Egypt 1922; spez. zu Thoth als Verfasser der Tempelbücher: S. 88-91. 
heißt natürlich in diesem Fall kultische, sakramentale Wirksamkeit, "verklärende", zu-einem-Lichtgeist-machende, erleuchtende Kraft. Das Sinnbedürfnis verlangt den modernen Text. Das alte Totenbuch genügt nicht mehr. Das Wahrheits- oder Wirksamkeitsbedürfnis verlangt den heiligen Text, bei dem es nicht auf menschliches Verstehen, sondern göttliches Angesprochenwerden ankommt. Göttliche Verfasserschaft ist die Lösung dieses Problems. Die Dokumente vom Atmen hat Isis selbst geschrieben, als es um die Wiederbelebung ihres Gatten Osiris ging.

Alle Handlungen, die im Kult vollzogen werden - das ist der Grundgedanke dieser Idee einer götterweltlichen Angemessenheit bzw. "Kompatibilität" und sakramentalen Wirksamkeit liturgischer Rezitationen - werden auch in der Götterwelt vollzogen. Wie im Himmel, so auf Erden, lautet das Prinzip. ${ }^{21}$ Hätte Isis nicht ihren Gatten Osiris mit ihren Klagen erweckt und mit ihren Verklärungen zu einem machtvollen unsterblichen Geistwesen gemacht, dann wären auch alle irdisch-kultischen Handlungen und Rezitationen in dieser Hinsicht zwecklos. Würde der Sonnengott nicht Tag für Tag den Chaosdrachen Apopis besiegen, der ihn mit Finsternis und Stillstand bedroht, dann hätten auch die Schutzriten, die täglich in den Tempeln zur Abwehr der inneren und äußeren Feinde Pharaos und zur Erhaltung der Ordnung und Wohlfahrt des Staates durchgeführt wurden keine Wirkung. Daher faßt man diese Texte auch unter einem sehr bezeichnenden Gattungsnamen zusammen: sie heißen $b^{3} w R^{c} w$, “die Machterweise des Re", weil man annimmt, daß sich in ihrer Rezitation die Macht des Sonnengottes selbst ereignet, mit der er in seinem täglichen Umlauf um die Erde die kosmischen Widerstände überwindet. ${ }^{22}$

Man denkt sich den Kosmos und die ihn verkörpernde Götterwelt als ein Drama und versteht die kultischen Handlungen als Abbildungen götterweltlicher Interaktion. Der Kult wird also nicht im Sinne einer Kommunikation zwischen Mensch und Gott vollzogen, sondern als die Inszenierung eines inner-götterweltlichen Dramas zwi-

${ }^{21} \mathrm{Zu}$ "descensio" und "translatio" als den Grundprinzipien des ägyptischen Kults im Sinne einer irdischen Abbildung himmlischer Vorgänge vgl. Corpus Hermeticum, Asclepius 23ff. und dazu Verf., Ägypten, 50-67.

${ }^{22} \mathrm{Zu}$ den $b^{3} w-R^{c} w$ vgl. Gardiner, $\mathcal{F} E A$ 24, 166, 168; Blackman, JEA 29, 22 Anm. 7; E.A.E. Reymond, CdE 47 (1972), 124; Verf., Liturgische Lieder an den Sonnengott, Berlin 1969, 222 Anm. 171; Stricker, Brief van Aristeas, 52-53; Schott, HdO I2, Literatur, 221 f.; D.B. Redford, Pharaonic King Lists, Annals and Day-Books. A Contribution to the Study of the Egyptian Sense of History, Mississauga 1986, 92, 215 f. Anm. 52 
schen Gott und Göttern. Dieses Prinzip war übrigens auch Jamblich noch vollkommen bewußt und er wird nicht müde, es in immer neuen Formulierungen zu beleuchten, um den Vorwurf zu entkräften, der Theurg wolle den Göttern drohen, sie zwingen oder sonstwie nach seinem Willen beeinflussen. Seine Argumentation beruht auf dem Gedanken, daß er ja nicht als Mensch den Göttern gegenübertritt, sondern von einer ekstatischen Position aus spricht, die am Göttlichen Anteil hat. Er zieht die Götter nicht zu sich herab, sondern vielmehr sich zu den Göttern empor "denn eine solche Anrufung zieht ja keineswegs die Unbeeinflußbaren und Reinen in die Sphäre der Affekte und Unreinheit hinab, sondern macht vielmehr im Gegenteil uns Menschen, die wir durch die Geburt den Affekten unterworfen wurden, rein und über den Affekt erhaben" (I 12). ${ }^{23}$ Daher besteht er darauf, "daß das Wirken der Götter nicht gewirkt wird, während zwei einander gegenüberstehende und von einander verschiedene Parteien einander gegenüberstehen (Mensch und Gott), sondern daß vielmehr diese Art göttlichen Wirkens in Übereinstimmung, Einheit und Einverständnis vollbracht wird" (IV 3). ${ }^{24}$ "Der Theurg gibt den kosmischen Mächten infolge der Kraft der geheimen Symbole seine Befehle nicht mehr als Mensch und auch nicht mehr als über eine nur menschliche Seele verfügend, sondern erteilt, als gehöre er jetzt zur Rangklasse der Götter, Befehle, die kräftiger sind als seine ihm tatsächlich zustehende Wesenheit" (VI 6). ${ }^{25}$ Deutlicher kann man den Grundgedanken auch der altägyptischen Ritualistik nicht umschreiben. Dieses "theurgische" Prinzip gilt für die Handlung und es gilt insbesondere für die von diesem Handeln nicht zu trennende Sprache. In den die Handlungen begleitenden Rezitationen liegt die verwandelnde, verklärende Kraft der Begehung. Deshalb ist stets der Priester mit der Schriftrolle dabei. Er verwaltet die sprachliche Seite der Begehung, die Rezitation, die im Mund des Priesters und im Augenblick der kultischen Handlung zur Götterrede wird. Wenn der Priester spricht, spricht ein Gott zum Gott und die Worte entfalten ihre verwandelnde, performative und präsentifikatorische Kraft. Das ist die Aufführung. Was der Vorlesepriester in der Hand hält, ist die Partitur.

Die heilige Rezitation ist also ihrem Sinn und ihrem Wesen nach

\footnotetext{
${ }^{23}$ Nach Hopfner, 26.

${ }^{24}$ Hopfner, $121 \mathrm{f}$.

${ }^{25}$ Hopfner, $159 \mathrm{f}$.
} 
Götterrede, gespeichert im Medium der Schrift und realisiert im Kontext des kultischen Rollenspiels. Der Priester äußert sie nicht in eigener Sache, er tritt damit nicht als Mensch vor ein Götterbild. Er schlüpft vielmehr in eine Rolle im Zusammenhang einer götterweltlichen "Konstellation". Der Kosmos, die Wirklichkeit ist aus solchen "Konstellationen" aufgebaut. Es sind die Strukturelemente, aus denen sich das Ganze der sich unablässig ereignenden "Welt" zusammensetzt. Die Sprache vermag sie zu beschreiben und narrativ zu verknüpfen: so entstehen die Mythen. Sie vermag aber auch gewissermaßen intra-konstellativ die bei diesem Zusammenwirken gesprochenen Worte zu artikulieren und dramatisch zu gestalten: so entstehen die Kultrezitationen.

Die Schrift dient dem Zweck, den heiligen Text vor Veränderung zu schützen und ihn, von Rezitation zu Rezitation, in seinem Wortlaut zu bewahren. Damit ist aber der Schutzbedürftigkeit des Heiligen Textes noch keineswegs Genüge getan. Zur Schrift tritt als zweite Schutzmaßnahme die Geheimhaltung hinzu. Das Geheimnis gehört in Ägypten zum Begriff des Heiligen. Das Heilige ist für den Ägypter eo ipso geheim. Wenn die Schutzzonen um das Heilige niedergerissen werden, fällt der Himmel auf die Erde herunter, wandelt sich Meer- in Süßwasser und wird alsobald ausgetrunken, steigen Flammen aus dem Ozean auf und verzehren das Feuer, trocknen die Flüsse und Seen aus. ${ }^{26}$

\section{Unio liturgica}

Als Handelnder schaltet sich der Offiziant ein in das götterweltliche Handlungsgefüge, das den Kosmos zusammen- und in Gang hält. Als Sprechender stimmt er ein in den götterweltlichen Diskurs. Dieses Prinzip möchte ich mit einem Begriff, den ich dankbar dem Judaisten Peter Schäfer entlehne, "unio liturgica" nennen. ${ }^{27}$ Im kultischen Sprechen tritt der Redende ein in eine götterweltliche Kon-

${ }^{26}$ Vgl. hierzu Porphyrius in Jamblichs Wiedergabe, De mysteriis VI.5: "Denn der Rezitierende droht, das Firmament zu zerschmettern, die Geheimnisse der Isis offenbar zu machen, das im Abgrunde (der Welttiefe) Verborgene aufzuzeigen, die Barke zum Stehen zu bringen, die Glieder des Osiris dem Typhon hinzustreuen oder überhaupt etwas dieser Art zu tun" (nach Hopfner, 159).

${ }_{27}$ P. Schäfer, Der verborgene und der offenbare Gott, Tübingen 1991, $160 \mathrm{ff}$. Auch in der frühjüdischen merkaba-Mystik geht es darum, in die himmlische Liturgie einzustimmen. Das wird hier aber nicht mehr kollektiv-kultisch erreicht, durch den genauen Vollzug des Tempelrituals, sondern individuell-mystisch, durch die Himmelsreise des 
stellation und spielt die Rolle göttlicher Aktanten. Er identifiziert sich mit ihnen oder, wie es ägyptisch heißt, "vereint sich mit ihnen":

Ich habe der Sonne Hymnen gesungen, ich habe mich mit den Sonnenaffen vereint, ich bin einer von ihnen.

Ich machte mich zum Genossen der Isis und stärkte ihre Zauberkraft ${ }^{28}$

Wer als Priester einen Sonnenhymnus spricht, im Rahmen der vorgeschriebenen kultischen Begehungen, der vereint sich mit den götterweltlichen Adoranten des Gottes und wird für die Dauer der Begehung "einer von ihnen". ${ }^{29}$ Der Hohenpriester Nebwennenef sagt:

Ich befriedige ihn (den Sonnengott) Tag für Tag,

ich singe Loblieder wie die Sonnenaffen,

ich verehre ihn wie die "Seelen" von Buto und Hierakonpolis"30

Alle kultischen Zeremonien sind von diesem identifikatorischen Typus. Es geschehen da wahrhaftig große Dinge. Im Kontext der oben zitierten Verse des 100. Totenbuchkapitels ist auch von anderen Ritualen die Rede:

Ich habe den Phönix zum Osten übergefahren und Osiris nach Busiris.

Ich habe die Grüfte des Hapi geöffnet

und den Weg der Sonne freigehalten.

Ich habe den Sokar auf seinem Schlitten gezogen

Adepten (jored merkaba). "Ziel der Himmelsreise ist weniger die Schau Gottes auf seinem Thron als vielmehr die Teilhabe am kosmischen Lobpreis ... Seine Himmelsreise dient dem Zweck, die irdische Gemeinde in die himmlische Liturgie einzubeziehen, diese zu einem wahrhaft kosmischen, Himmel und Erde, Engel und Menschen umfassenden Ereignis werden zu lassen" (S. 160). Präziser könnte man auch die Aufgabe des altägyptischen Sonnenpriesters nicht beschreiben. Auch sein Lobpreis stimmt in die himmlische Liturgie ein, und auch hier liegt der Sinn solchen Einstimmens darin, Himmel und Erde zueinander in Beziehung zu setzen und eine kosmische Harmonie herzustellen. Mystik und Magie erweisen sich hier wie in der Religion der Papyri Graecae Magicae als gewissermaßen "privatisierte" Nachfolgeinstitutionen des ägyptischen Tempelkults.

${ }_{28}$ Totenbuch, Kapitel 100. Hornung, Das Totenbuch der Agypter, Zürich 1979, 198 f. H. te Velde, "Some Remarks", 129.

${ }^{29}$ Vgl. Totenbuch 81B (Hornung, 168):

Ich kenne den Spruch derer, die mit diesen Göttern, den Herren des Totenreichs, sind.

Ich bin einer von euch.

Laßt mich doch die Götter schauen, welche die Unterwelt leiten, gebt mir einen Platz im Totenreich!

${ }^{30}$ Grab Theben 157, Wörterbuch Zettel <1112>; J. Zandee, JEOL 18, 1964, 253. 
und die Große im rechten Augenblick gestärkt. . . .

Ich habe das Seil festgebunden,

ich wehrte den Apopis ab und zwang ihn zum Rückzug.

Re hat mir seine Arme entgegengestreckt

und seine Mannschaft wird mich nicht zurückweisen. ${ }^{31}$

Hier spricht jemand, der an verschiedenen Festen, Riten und Prozessionen teilgenommen hat. Er darf sich dadurch als ein Mitglied götterweltlicher Konstellationen ausgeben. Das ist der Sinn der ägyptischen "Mysterien". Es handelt sich dabei nicht um Geheimgesellschaften, um Gemeinschaften, zu deren Glauben man sich bekennen und in die man sich einweihen lassen kann. Es handelt sich ganz einfach um den normalen, offiziellen ägyptischen Kult. Er beruht auf dem Prinzip der götterweltlichen Identifikation, der "unio liturgica". Aus diesem Prinzip folgen mit einer gewissen logischen Konsequenz zwei Motive, die diesen Kult mit dem antiken Mysterienwesen verbinden: Geheimhaltung und Einweihung.

Re hat ihn (den Sprecher) eingeführt in seine Barke:

er hat die Heiligkeit Dessen in seiner Umringlerschlange gesehen.

Er hat Re betrachtet, nämlich die drei Gestalten, die er annimmt in der Ausdehnung des Lichtglanzes.

Er hat ihn angebetet bei seiner Geburt am Morgen

in jenem seinem Namen "Chepre",

er hat ihn gepriesen am Mittag

in jenem seinem Namen "Re",

er hat ihn besänftigt am Abend

in jenem seinem Namen "Atum". 32

Wie schön ist es, zu schauen mit den Augen,

wie schön ist es, die Wahrheit zu hören mit den Ohren!

... Osiris NN hat nicht weitergesagt, was er gesehen hat,

Osiris NN hat nicht berichtet, was er gehört hat im Haus der Mysterien: den Jubel für $\mathrm{Re}$

und den Gottesleib des Re beim Queren des Nun

unter denen, die den Gottes-Ka befriedigen mit dem, was er liebt. ${ }^{33}$

Ein unlängst publizierter kleiner Sonnenhymnus auf einem Sarg der Spätzeit in Cambridge gibt sich als Rede des "Kollegium im Urwasser", worunter wohl die acht Urgötter zu verstehen sind, auf deren Anbetung des Sonnengottes wir in anderem Zusammenhang noch zurückkommen werden:

\footnotetext{
${ }^{31}$ Hornung, Totenbuch, $198 \mathrm{f}$.

32 Totenpapyrus Louvre 3292, ed. Nagel, BIFAO 29, 47.

${ }^{33}$ TB 133, 14-15 (Nu).
} 
Re anbeten bei seinem Aufgang im östlichen Lichtland seitens des Großen Kollegiums im Urwasser.

$\mathrm{Zu}$ sprechen: Sei gegrüßt, Re, Tag für Tag,

Chepre, der von selbst entstand,

mit vielen Namen und vielen Gesichtern, der zu Schiff fährt ohne zu ermüden.

Komm doch, Re, in Frieden!

Richte dich auf: dein Feind ist gefallen. ${ }^{34}$

Die Reden der Götter, also z.B. die Hymnen, die das "Große Kollegium" oder die "Sonnenaffen" an den Sonnengott richten, sind "geheime" Worte. Sie kennt nur der Eingeweihte. Diese Einweihung kennzeichnet nach ägyptischer Vorstellung die Rolle des Königs. Natürlich waren es in der geschichtlichen Wirklichkeit nicht die Könige, sondern die Priester, die als Träger, Verwalter, Überlieferer und Anwender dieses Wissens fungierten. Der Begriff "König" bezeichnet eine Institution, eine sakrale Funktion, die vom Priester und nicht vom wirklichen König wahrgenommen wird. Der Priester spielt den König, um im Rahmen des kultischen Dramas den Gott spielen zu können. Von diesem theoretischen "König" heißt es nun in einem für diese Konzeption kultischen Wissens und Sprechens zentral wichtigen Text:

Der König kennt

diese geheime Rede, die die 'östlichen Seelen' sprechen, wenn sie Jubelmusik machen für den Sonnengott

bei seinem Aufgang, seinem Erscheinen im Horizont

und wenn sie ihm die Türflügel öffnen

an den Toren des östlichen Horizonts,

damit er zu Schiff dahinfahren kann auf den Wegen des Himmels.

Er kennt ihr Aussehen und ihre Verkörperungen,

ihre Wohnsitze im Gottesland.

Er kennt ihre Standorte

wenn der Sonnengott den Weganfang beschreitet.

Er kennt jene Rede, die die Schiffsmannschaften sprechen,

wenn sie die Barke des Horizontischen ziehen.

Er kennt das Geborenwerden des Re

und seine Verwandlung in der Flut.

Er kennt jenes geheime Tor, durch das der Große Gott herauskommt, er kennt den, der in der Morgenbarke ist, und das große Bild in der Nachtbarke.

${ }^{34}$ M. el Alfi, "A Sun Hymn in the Fitzwilliam Museum", in: Varia Aegyptiaca 8.1, 1992, 3-5. 
Er kennt seine Landeplätze am Horizont

und deine Umläufe in der Himmelsgöttin. ${ }^{35}$

Dieser Text zählt auf, was der König alles wissen muß für eine einzige, wenn auch entscheidende Handlung: die Anbetung des Sonnengottes am Morgen. Er kennt die Natur des kosmischen Vorgangs, seine Phasengliederung, seine szenisch-konstellative Ausgestaltung und seine Heilsbedeutung als Wiedergeburt, er kennt die beteiligten Wesen, ihre Handlungen, ihre Reden, ihre Lebensumstände, und er kennt den räumlichen Rahmen des Geschehens, Himmelstore, Barken, Landeplätze, Steuergeräte. Er muß das alles genau kennen, um sich mit seiner anbetenden Rede wirkungsvoll in den kosmischen Prozeß einschalten zu können.

Einen entsprechenden Text gibt es auch für die Anbetung des Sonnengottes am Abend. ${ }^{36}$ In diesem Text spielt die Kenntnis der götterweltlichen Liturgie eine ganz besonders prominente Rolle:

Veranlaßt wird, daß die Götter zur Ruhe gehen in der Erde durch die geheime Rede in seinem (des "Königs") Munde.

"O Heiliger in der Nachtbarke, Herr des Lebens im Westland!"

Heiligkeit und Göttlichkeit werden gegeben dem Großen Ba (durch) die Gottesworte.

Der erscheint mit seinem Einauge, der Herrin an der Spitze der beiden Barken, entsprechend den Lobgesängen, die ihm die Westlichen des Himmels singen in ihren Erscheinungsformen:

"Der ausgestattet ist mit seinen Formen, der Jahrmillionen verbringt, Re möge ruhen, möge ruhen im Innern der Erde", so singen sie für dich, "Der ausgestattet ist mit seinem Göttlichen Auge, das Falkenbild in der Nachtbarke, der Herr der Heiligkeit im westlichen Lichtland!" Die Menschen leben auf, wenn sie ihn sehen, die Götter jubeln, wenn sie seine Schönheit erblicken.

Oh juble, Re, über die Preisungen des Königs, wenn er Re verehrt mit Hymnen.

${ }^{35}$ Aus einem Kulttheologischen Traktat über den "König als Sonnenpriester", ed. J. Assmann, Der König als Sonnenpriester, Glückstadt 1970; Maria Carmela Betrò, I Testi solari del Portale di Pascerientaisu (BN2) (Università degli Studi di Pisa, Missioni Archeologiche in Egitto, Saqqara III) (Pisa 1990).

${ }^{36}$ Die Wiedergewinnung dieses Textes ist das besondere Verdienst von M.C. Betrò, s. die vorhergehende Anmerkung. 
Wie am Morgen mit "pavianischen" Lobpreisungen, so begrüßt der Sonnenpriester am Abend den Sonnengott mit "schakalischen" Hymnen. Natürlich gebraucht er dabei keine Tier- oder sonstige FremdSprache, sondern das zur Kult- und Göttersprache gewordene klassische Ägyptisch. Die pavian- und schakalsgestaltigen "Seelen" des Ostens und Westens sind keine Tiere, sondern eine Art Engel. Sie gehören zur Götter-, nicht zur Tierwelt. ${ }^{37}$ In ihre Lobpreisungen stimmt der irdische Sonnenpriester mit seinen Sonnenhymnen ein.

Hier gilt es nun eine wichtige Unterscheidung zu berücksichtigen. Diese Vorstellung einer geheimen Preisung in Göttersprache scheint keineswegs für die gesamte ägyptische Sonnenhymnik zu gelten. Vielmehr hat man hier offenbar eine esoterische und eine exoterische Tradition zu unterscheiden. Aus dem alten Ägypten sind uns Hunderte von Sonnenhymnen erhalten. Sie stehen zumeist auf den Wandungen der Grabeingänge und zeichnen die Worte auf, mit denen der Grabherr morgens und abends, aus seinem Grabe aus- und eintretend, die Sonne anbeten wollte. Andere Hymnen stehen auf Stelen, die im, über oder auch vor dem Grab aufgestellt waren. Alle diese Texte waren zugänglich angebracht und bilden die exoterische Form der ägyptischen Sonnenhymnik. Sie geben sich nicht als Aufzeichnung geheimen Wissens.

Es gibt aber eine Überlieferungsform, die man ganz eindeutig als exklusiv und esoterisch einzustufen hat. Das sind die Bilder und Texte, mit denen die Königsgräber im Neuen Reich ausgeschmückt waren. Denn diese Gräber waren nicht zugänglich, sondern im Gegenteil hermetisch versiegelt und strengstens bewacht. In ihnen fand kein Kult statt, im Unterschied zu den Privatgräbern. Der Kult hatte seinen Ort in den Totentempeln, die räumlich weit von den Gräbern getrennt errichtet wurden. In den Königsgräbern finden wir nun eine Literatur, die genau jenes Wissen kodifiziert, das in den oben zitierten Traktaten dem "König" als Priester des Sonnengottes zugeschrieben wird. ${ }^{38}$ Hier stehen die Hymnen, die die Jenseitigen an den Sonnengott richten, hier sind alle Handlungen, alle dramatis personae

${ }^{37}$ Dieser Unterschied wird von H. te Velde in seinem wichtigen Aufsatz über die "mysterious language" der Sonnenaffen nicht ausreichend berücksichtigt.

${ }^{38}$ E. Hornung, Agyptische Untenveltsbücher, Zürich und München ${ }^{2} 1984$, vgl. Hornung, "Auf den Spuren der Sonne. Gang durch ein ägyptisches Königsgrab", in: Eranos Jahrbuch 1981, 431-475 und H. Brunner, "Die Unterweltsbücher in den ägyptischen Königsgräbern", in: G. Stephenson (Hg.), Leben und Tod in den Religionen. Symbol und Wirklichkeit, Darmstadt 1980, 215-228. Allgemein zu den Königsgräbern s. E. Hornung, Tal der Könige. Die Ruhestätte der Pharaonen. Zürich 1982. 
und alle Lokalitäten genau beschrieben, in deren Rahmen sich das götterweltliche Drama abspielt. Der wichtigste, älteste und gewissermaßen klassische Text dieser Gattung, das "Amduat" 39 hat folgenden Titel:

Zu kennen die Wesen der Unterwelt,

zu kennen die geheimen Wesen,

zu kennen die Tore und die Wege, auf denen der Große Gott (der

Sonengott) wandelt,

zu kennen, was getan wird,

zu kennen, was in den Stunden ist und ihre Götter,

zu kennen den Lauf der Stunden und ihre Götter,

zu kennen ihre Verklärungssprüche für Re,

zu kennen, was er ihnen zuruft,

zu kennen die Gedeihenden und die Vernichteten. ${ }^{40}$

Es handelt sich hier also um ein Buch, das in erster Linie Wissen kodifizieren, systematisieren und vermitteln will. Das Wort Wissen oder Kennen wird im Titel neunmal wiederholt. In diesen Büchern haben wir die Kodifikationen des magischen Wissens vor uns, das nach Ansicht der Ägypter zur In-Gang-Haltung des (nächtlichen) Sonnenlaufs notwendig ist. Als Grabdekoration dienen sie dem König dazu, sein mitwirkendes Teilnehmen und seine Teilhabe am Gelingen des Sonnenlaufs im Jenseits fortzusetzen. Aus der Art dieser Wiederverwendung, der wir die Kenntnis dieser Literatur verdanken, können wir schließen, daß es sich dabei um einen äußerst exklusiven, streng gehüteten Wissensvorrat gehandelt haben muß. Denn im Neuen Reich (16.-12.Jh.) kommen die Kosmographien so gut wie ausschließlich in Königsgräbern vor. Der Charakter eines hermetischen Geheimwissens wird in ihnen selbst auch immer wieder betont. "Die geheimnisvolle Schrift der Unterwelt" nennt sich das Amduat, "die nicht gekannt wird von irgendwelchen Menschen außer vom Erlesenen". ${ }^{41}$

Letztlich geht es bei diesem Wissen um Teilnahme und Teilhabe. Magie und Mystik hängen ganz eng zusammen. ${ }^{42}$ "Wer das weiß, ist

${ }^{39}$ Ed. E. Hornung, Das Amduat. Die Schrift des Verborgenen Raumes, 3 Bde, Wiesbaden, 1963-67; Übers. Hornung, Unterweltsbücher, 57-194.

${ }^{40}$ Hornung, Unterweltsbücher, 59.

41 Hornung, a.a.O., 193.

${ }^{42}$ Auf den Zusammenhang von Magie und Mystik haben wohl am nachdrücklichsten Gershom Scholems Interpretationen der kabbalistischen Traditionen aufmerksam gemacht. Auch bei den ägyptischen Unterweltsbüchern handelt es sich um eine Art Kabbalah, aber sozusagen eine "Staats-Kabbalah", der zur vollen Entfal- 
ein Ebenbild des Großen Gottes" heißt es ausdrücklich im Amduat. ${ }^{43}$ Die Teilhabe am Wesen der Gottheit wird aber nicht durch Versenkung und Meditation des Individuums erreicht, sondern durch die rituelle Inszenierung dieses Wissens in der sozialen Rolle eines bevollmächtigten Priesters. Der Priester handelt und spricht, wie schon gesagt, nicht in eigener Sache sondern in gesellschaftlichem Auftrag. Um diesen Auftrag wahrzunehmen, identifiziert er sich mit einer götterweltlichen Rolle. Nur als Mitglied der Götterwelt kann er fördernd in das götterweltliche Drama eingreifen.

Den wichtigsten Wissensgegenstand, den diese Geheimliteratur kodifiziert, bildet die Liturgie der jenseitigen Wesen, die Worte, die sie an den Sonnengott richten. Diese Liturgie der Unterweltlichen durch Textbeispiele zu illustrieren, würde hier zu weit zu führen. Ich verweise auf Hornungs handliche Textzusammenstellung der "Ägyptischen Unterweltsbücher," ${ }^{44}$ die schon beim flüchtigen Durchfliegen eine Fülle eindrucksvoller Beispiele liefert. Diese Texte bestehen zum Großteil aus direkter Rede, und ein großer Teil dieser Reden wiederum besteht in Preisungen, die von den Unterweltlichen an den Sonnengott gerichtet werden.

Im Umkreis dieser Literatur, die man in einem gewissen Sinne, der nach allem Gesagten hoffentlich hinreichend klar geworden ist, als magisch, mystisch und esoterisch einstufen kann, stößt man auch auf eine Reihe von Sonnenhymnen, die in den oben genannten Gräberhymnen nicht vorkommen und die sich als götterweltliche Anbetungen verstehen. Wer sie kennt und rezitiert, der stimmt in diese jenseitige Anbetung ein und vereint sich im Sinne der unio liturgica mit den götterweltlichen Adoranten. Der oben zitierte Traktat über den König als eingeweihten Priester des Sonnengottes gehört zu

tung ihrer mystischen Komponenten die Individualität des Subjekts fehlt. Wer hier zum "Ebenbild des Großen Gottes" wird ist der König und in seiner Stellvertretung der Sonnenpriester, immer als bevollmächtigter Repräsentant der Menschheit insgesamt, die es in solcher assimilatio dei mit den kosmogonischen Kräften in Einklang $\mathrm{zu}$ bringen gilt. Allerdings tritt auch in der frühjüdischen Mystik der Adept (jored merkavah) als Repräsentant der Gemeinde auf, vgl. P. Schäfer, Der verborgene und offenbare Gott, Tübingen 1991, 45-48.

${ }^{43}$ Diese Aussagen hat E.F. Wente, "Mysticism in Ancient Egypt?", in: Journal of Near Eastern Studies 41, 1982, 161-179, analysiert und dabei die gottgleichmachende Heilseffizienz des in diesen Büchern vermittelten magischen Kosmos-Wissens gebührend hervorgehoben. Gegen seine einseitige Interpretation dieser Aussagen als "mysticism" sind die in der vorhergehenden Anmerkung dargelegten Einschränkungen geltend zu machen.

${ }^{44}$ E. Hornung, Unterweltsbücher. 
einem Zyklus von Texten, die das Dekorationsprogramm der Sonnenheiligtümer des Neuen Reichs bilden. ${ }^{45} \mathrm{Zu}$ diesem Zyklus gehört auch der folgende Hymnus, der das Prinzip der unio liturgica, der Einstimmung des kultischen Lobpreises in den Lobpreis der götterweltlich-jenseitigen Anbeter des Sonnengottes, explizit zum Ausdruck bringen: ${ }^{46}$

Sei gegrüßt mit dem, was dir dein Auge sagt,

das dir den Weg der Ewigkeit bahnt.

Sei gegrüßt mit dem, was dir deine Sonnenscheibe sagt,

wenn sie zu dir aufsteigen läßt die, die in Schrecken vor dir sind.

Sei gegrüßt mit dem, was die Mesektet-Barke zu dir sagt,

wenn sie dahinfährt [in günstigem Segelwind]-

Sei gegrüßt mit dem, was ihre Gefährtin, die Me'andjet-Barke zu dir sagt, wenn ihre Schwester anhält.

Sei gegrüßt mit dem, was dein Stab zu dir sagt, wenn er sich ganz deiner Faust gesellt hat.

Sei gegrüßt mit dem, was deine Mannschaft zu dir sagt, wenn der Falke ihre Bilder hervorgebracht hat.

Sei gegrüßt mit dem, was die Lichtland-Bewohner zu dir sagen, die Wächter am Ufer des Himmels!

Sei gegrüßt mit dem Spruch der Treidler,

die das Ende des Zugseils ergreifen.

Sei gegrüßt mit dem, was die Unterweltsbewohner zu dir sagen, wenn sie deine beiden Barken auf ihren vier Wegen begleiten.

So wendet sich auch eine Anrufung im gräco-ägyptischen Leidener Zauberpapyrus an den Sonnengott:

Tritt ein, erscheine mir, Herr, daß ich dich anrufe, wie dich die drei Hundskopfaffen anrufen, welche in symbolischer Gestalt deinen heiligen Namen nennen ${ }^{47}$

Der Text der Königin Nedjemet zählt die Mitglieder der nächtlichen Konstellationen auf, in denen der Sonnengott die Fahrt durch die Unterwelt vollzieht: das "Auge" (die Stirnschlange des Gottes, das Abzeichen seiner Herrschaft), seine Sonnenscheibe, die mit ihrem Licht zugleich auch den "Schrecken", die Ehrfurcht vor dem Gott, verbreitet, seine beiden Barken, sein Stab, den er in der Hand

${ }^{45}$ S. dazu Verf., "Das Dekorationsprogramm der königlichen Sonnenheiligtümer des Neuen Reichs nach einer Fassung der Spätzeit”, in: ZÄS 110, 1983, 91-98.

${ }^{46}$ Verf., Re und Amun. Die Krise des polytheistischen Weltbilds im Agypten der 18.-20. Dynastie, OBO 51, Fribourg 1983, 27-29. Medinet Habu VI, 422A, 36 ff.; J.C. Goyon, in: R.A. Parker et al., The Edifice of Taharqa bei the Sacred Lake of Karnak, Providence 1979, 44 f., Tf. 35-37; Totenbuch der Königin Nedjemet, pBM 10541. Alle drei Varr. sind bei Goyon im Hieroglyphentext zusammengestellt.

47 PGM IV 999-1005; Reitzenstein, Poimandres, 27; Verf., Liturgische Lieder, 345. 
hält, seine Mannschaft, die ihn in der Nachtbarke durch die Unterwelt begleitet, die Bewohner des "Lichtlands", der Grenzregion zwischen Diesseits und Jenseits, die schakalsgestaltigen "Seelen des Westens", die die Sonnenbarke durch die Unterwelt treideln und die Bewohner der Unterwelt. Alle diese Mitwirkenden in dem nächtlichen Drama reden den Gott an, der das Zentrum ihrer Konstellationen bildet, und artikulieren im Medium der Sprache ihre Rolle im kosmischen Geschehen. Der irdische Sonnen-Priester kennt diese Reden und vermag mit seiner eigenen Rede in sie einzustimmen und auf diese Weise ebenfalls zum Mitglied der nächtlichen Konstellationen zu werden. Die Königin Nedjemet, die diesen Hymnus zusammen mit anderen Texten gleicher Herkunft in ihr Totenbuch aufnehmen konnte, weil sie als Königin und Gemahlin des in der Zeit des Gottesstaats als König amtierenden Hohepriesters Zugang zu esoterischem Schriftgut hatte, stellt diese Kenntnis der jenseitigen Liturgie und ihre dadurch ermöglichte Mitgliedschaft in der jenseitigen Gemeinde, also das Prinzip der unio liturgica, noch einmal eigens heraus:

Osiris Nedjemet, gerechtfertigt, kennt jene Worte, die die östlichen Seelen sprechen;

Osiris Nedjemet ist inmitten deines Kollegiums,

Osiris, und tritt ein in die Mannschaft des Re, Tag für Tag. ${ }^{48}$

Das Prinzip der unio liturgica, wie es sich nach den angeführten Texten im ägyptischen Sonnenkult darstellt, läßt sich auf folgende drei Punkte bringen:

Eingeweihtsein:- ich kenne die Worte, mit denen die Jenseitigen dich preisen (Wissen)

Priesterlicher Kultvollzug:-ich preise dich mit diesen Worten (Handeln)

Götterrolle -ich gehöre zu der jenseitigen "Gemeinde" (Identität).

Im Kult ermöglicht dieses Prinzip die Kommunikation mit der Götterwelt, die Einbindung der kultischen Vollzüge in das kosmische Geschehen. Im Totenglauben aber ermöglicht es den Eintritt des Verstorbenen in die Götterwelt und die Verwandlung in eine götterweltliche, unsterbliche Identität in den Konstellationen des Sonnenlaufs. Im Kult geht es um ein Ritual zur Inganghaltung der Welt, im Totenglauben aber geht es um den individuellen Menschen, seine

${ }^{48}$ Re und Amun, 52. 
Gottesnähe und Unsterblichkeit. Der ägyptische Totenglauben transformiert die kultisch-magischen Prinzipien kosmischer Ein- und Mitwirkung in das gewissermaßen "mystische" Prinzip der individuellen Einswerdung mit dem Göttlichen, der unio liturgica. In diesem Rahmen vermittelt die Kenntnis der esoterischen Kulttexte Göttlichkeit (Zugehörigkeit zur Götterwelt) und Unsterblichkeit. Es handelt sich aber dabei um einen sekundären Rahmen. Der eigentliche "Sitz im Leben" des Gedankens der unio liturgica ist kultisch und hat mit individueller Mystik nichts zu tun.

Ein noch viel entscheidenderer Schritt in Richtung einer individuellen Gottesnähe wird vollzogen, wenn das Prinzip der unio liturgica, die Einweihung und Einstimmung in den Lobpreis der Himmlischen, nicht erst im Totenkult, sondern schon bei der Einweihung der Lebenden Anwendung findet. "Wenn die verklärte Königin", so kommentieren R. Merkelbach und M. Totti die oben zitierten Sätze aus dem Totenbuch der Königin Nedjemet, "die Rede der Götter kennt, dann ist sie schon zu Lebzeiten eingeweiht worden, nicht anders als der Initiand unseres griechischen Textes" ${ }^{49}$ Genau dies ist die Frage. Den Text der Königin Nedjemet zitieren R. Merkelbach und M. Totti, um den ägyptischen Traditionshintergrund der Leidener "Kosmopoiie" herauszuarbeiten. Dort geht es um die Anrufung des Urgottes und Weltschöpfers "in allen Stimmen und Sprachen", worunter, genau wie in den altägyptischen Texten, die Worte gemeint sind, mit denen die Mitglieder der göttlichen "Sphäre des Seinigen" ihren Gott preisen. Genau wie der "König als Sonnenpriester" kennt "auch der Initiand unseres Textes (der Leidener Weltschöpfung) jene heiligen Worte, mit denen die Götter auf der Sonnenbarke den aufgehenden Sonnengott begrüßt haben" (S. 51) und: "ein Anbeter des Sonnengottes auf Erden stimmt in den Lobgesang ein, der dem Gott im Himmel entgegengebracht wird, und legitimiert sich, indem er die Rede der Götter wiederholt” (S. 52). Klarer läßt sich das Prinzip der unio liturgica in seinem altägyptischen Verständnis nicht umschreiben. Der einzige Unterschied zum griechischen Text liegt darin, daß der ägyptische Priester sich nicht legitimieren muß. Seine Legitimität liegt in seinem Amt, in der Institution des Kultes. Er spricht nicht als N.N. Anders steht es natürlich, wenn solche Texte in die "Totenliteratur" übernommen und einem Toten in den Mund

${ }^{49}$ Abrasax 3, 1992, 52. 
gelegt werden, der sie nicht in Ausübung eines priesterlichen Amtes, sondern als der verstorbene N.N. im Bedürfnis nach persönlicher Gottesnähe an den Gott richtet. Hier wird schon innerhalb der ägyptischen Tradition eine erste individualisierende Umdeutung der kultischen Rede unternommen. Einen weiteren Schritt solcher individualisierender Umfunktionierung bedeutet dann die Anwendung auf den lebenden N.N., der schon zu Lebzeiten in den Genuß persönlicher Gottesnähe kommen will. Diesen Schritt vollziehen die graecoaegyptischen Gebete und Weihezeremonien.

R. Merkelbach und M. Totti geht es um den allgemeinen ägyptischen Hintergrund. Der Gedanke der unio liturgica kennzeichnet aber eine spezifische Tradition. Er bildet nicht einfach das Grundprinzip der ägyptischen Hymnik, sondern findet sich vorzugsweise dort, wo es um exklusive Texte geht, Texte, die allem Anschein nach nur dem Eingeweihten zugänglich waren, "die nicht gekannt werden von irgendwelchen Menschen außer vom Erlesenen", wie es im Amduat heißt. Es handelt sich geradezu um das Leitmotiv einer kultischen Tradition, die ich aufgrund ihrer offenkundigen Exklusivität die "Mysterien" des Sonnenkultes genannt habe. ${ }^{50}$ Zwischen dem Anspruch eines Textes, die "geheime Rede" der götterweltlichen Adoranten wiederzugeben, und seiner eigenen Geheimheit besteht ein offenkundiger Zusammenhang.

Dieser Anspruch und diese Geheimheit sind keineswegs typisch für die ägyptische Hymnik im Allgemeinen. Diese ist im Gegenteil viel eher gekennzeichnet durch ein geradezu propagandistisches Pathos der Verkündigung, geboren aus dem Wunsch, werbend für den Gott einzutreten: "Ich will die Liebe zu dir verbreiten durch die Länder," 51 "ich will die Götter hören lassen und die Menschen wissen lassen von deiner Schönheit." " ${ }^{2}$ Das Grundmotiv der ägyptischen Hymnik ist eher exoterisch. Der Gedanke der "Einstimmung in den himmlischen Lobgesang", wie ihn R. Merkelbach und M. Totti unter Berufung auf das 1. Kapitel von Re und Amun und die dort zusammengetragenen Stellen herausstellen, kennzeichnet vielmehr eine klar abgrenzbare, besondere Tradition: die liturgische Preisung des Sonnengottes in den Sonnenheiligtümern. Mit diesem, und nur diesem Zweig der ägyptischen Sonnenhymnik verbindet sich eine ausgeprägt magische, theurgische

${ }^{50}$ Re und Amun, Kap. 1.

${ }^{51}$ Re und Amun, 184 m.Anm. 133.

${ }^{52}$ a.a.O., mit Anm. 134. 
Intention: die Vorstellung, mit der Rezitation zur Inganghaltung der Welt und zum Gelingen des kosmischen Dramas beizutragen.

\section{Der Lobpreis der acht Urgötter}

Das Prinzip der unio liturgica, der Übereinstimmung kultischen und götterweltlichen Lobpreises, findet sich jedoch auch außerhalb der Sonnenliturgie im engeren Sinne: in der kultischen Preisung des verborgenen Allgotts der "Thebanischen Theologie". ${ }^{53}$ Auch dort ist es mit Motiven und Überlieferungsformen verbunden, die in den Bereich des Geheimnisses und des Esoterischen weisen. Ich denke hier besonders an die beiden "Urgötterlieder" im Tempel von Hibis. Diesen Tempel ließ der Perserkönig Darius I. in der Oase el-Khargeh für Amun errichten und in einer für die damalige Zeit ungewöhnlichen, schon auf die griechisch-römische Tempeldekoration vorausweisenden Fülle beschriften. Unter den vielen Texten befindet sich auch eine Gruppe theologisch höchst anspruchsvoller Hymnen auf Amun-Re als Schöpfer- und Weltgott. Zwei dieser Hymnen sind schon 700 Jahre früher in Auszügen in einem magischen Papyrus bezeugt, gehen also auf das Neue Reich, vermutlich das 13.Jh.v.Chr. zurück. Diese beiden Hymnen geben sich als Loblieder, mit denen die acht Urgötter den Ur-, Schöpfer- und Weltgott Amun bei seinem ersten Erscheinen in ihrer Mitte gepriesen haben. Der eine trägt den Titel "Der große geheime Hymnus auf Amun-Re, den die Acht Urgötter gesprochen haben" und beginnt:

Sei gegrüßt, du Einer, der sich zu Millionen machte, der sich in Raum und Zeit ausdehnt ohne Grenzen, gerüstete Macht, die von selbst entstand, Uräusschlange mit gewaltiger Flamme, der Zauberreiche mit geheimer Gestalt, der geheime Ba dem Ehrfurcht erwiesen wird ${ }^{54}$

Der andere hat eine wesentlich längere Überschrift:

"Was die großen Acht der ersten Urzeit sprachen, als sie den Gott in ihrer Mitte verehrten, der zu Re geworden war,

[der Gott], der durch sich selbst entstand - seine Knochen waren aus Silber,

${ }^{53} \mathrm{Zu}$ diesem Gott und seiner Theologie vgl. Re und Amun, Kap. 5.

${ }^{54}$ Papyrus Mag. Harris IV,1-2 = Hibis 32,1, ÄHG Nr. 129, 1-6. 
seine Haut aus Gold,

seine Haare aus echtem Lapislazuli,

seine Zähne aus Türkis-......

indem sie ihn priesen bis zur Höhe des Himmels,

ihn anbeteten, der seine Kinder gebar,

die er heraufgeführt hatte aus dem Verborgenen,

indem sie für ihn musizierten auf ihren Harfen,

ihm Lobgesänge anstimmten für seinen $\mathrm{Ka}$ :

"Laßt uns ihm Hymnen singen als unserem Herrn!'”...

Sie erkannten Seine Majestät als ihren Herrn,

so wie er sich in allen diesen Werken ausgezeichnet hatte. Sein Name war, von den Bergen bis zum Meer:

Amun, der bleibt in allen Dingen",

dieser erlauchte Gott, der die Erde erschuf nach seinem Plan, Tatenen, der vor den Göttern ausgezeichnete,

der Greis der sich verjüngt und die Zeit durchläuft,

mit verborgenen Gesichtern, scharfen Augen und ausgedehnter Gestalt:

sein Leib ist der Wind,

der Himmel ruht auf seinem Haupt,

das Urwasser trägt sein Geheimnis ...." ${ }^{55}$

Dieser Hymnus weist nicht nur zurück ins 13.Jh., wo er als Zaubertext Verwendung fand, sondern auch voraus auf den Weltgott, den All-Einen "Peri-echon" der griechisch-ägyptischen Zauberpapyri, in denen sich fast identische Prädikationen finden, und auf das Corpus Hermeticum, in dem es ebenfalls ein Urgötterlied, einen Hymnus der ersten Achtheit gibt. ${ }^{56}$ Das Prinzip der unio liturgica ist hier anders zu deuten. Hier geht es nicht um kultische Mitwirkung im Drama der kosmischen Weltinganghaltung. Es geht nicht darum, mit dem Hymnus in eine der Konstellationen einzutreten, die an diesem Drama beteiligt sind. Man wird nicht zu einem der acht Urgötter, wenn man diesen Hymnus kennt und singt. Hier soll vielmehr der Text selbst als ein besonders authentischer Hymnus ausgezeichnet werden. Ein Hymnus, den die acht Urgötter, die Verkörperungen der Präexistenz angestimmt haben, gehört selbst der Präexistenz an. Hier stoßen wir auf das Prinzip der göttlichen Verfasserschaft, der den Hymnus als wahren, echten, wohlgefälligen und wirkungsvollen Text ausweist, wie es zum theurgischen Gebet gehört. Jetzt ist es nicht die Rede der jenseitigen Wesen, der götterweltlichen Konstellations-Mitglieder, die "geheim" genannt wird, sondern der Hymnus selbst ist

55 Papyrus Mag. Harris IV, 8-VI.4 = Hibis 33, ÄHG Nr. 130.

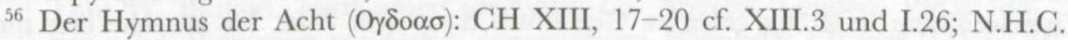
VI, 6.58-22-60,1; C.H.XIII, 21. 
geheim, die kultische Liturgie. Damit sind wir schon bei den graecoaegyptischen Zauberpapyri und bei theurgischen Gebet des Jamblich, mit dem einen Unterschied, daß diese "großen, geheimen Hymnen" noch zum offiziellen Kult der ägyptischen Staatsreligion gehören, während sie im spätantiken Ägypten das Schrifttum von Glaubensgemeinschaften im Rahmen eines religiösen Pluralismus bilden.

Ich komme zum Schluß und fasse zusammen. Das Motiv der unio liturgica findet sich in Ägypten im Kontext einer Überlieferung, die man im folgenden Sinne als "esoterisch" bezeichnen kann: sie ist geheim, d.h. strengen Zugänglichkeitsbeschränkungen unterworfen, und sie ist Gegenstand einer Einweihung. Der Sitz im Leben dieser Überlieferung ist der Sonnenkult, der als eine magische Veranstaltung zum Zwecke der Inganghaltung der Welt gedeutet werden muß. Im Dienste dieser kultischen Aufgabe übernimmt der Priester Götterrollen und rezitiert Götterrede. Dazu befähigt ihn seine Kenntnis der Schriften, in die er als Priester eingeweiht wird. ${ }^{57}$ Der Sammelname dieser Schriften ist $b^{3} w R^{c} w$ "die Machterweise des Re".

Die Götterrede der heiligen Texte gehört zu den Kultgeheimnissen, die nicht enthüllt werden dürfen, wenn anders die Welt nicht in ihrem Fortbestand gefährdet und dem Chaos Tür und Tor geöffnet werden soll. Insofern ist sie geheim und ist der Sonnenkult ein Mysterium. Solange es sich hier jedoch um den offiziellen ägyptischen Kult handelt, dürfen wir weder von Esoterik noch gar von Mystik sprechen. Mit Esoterik haben wir es erst zu tun, wenn diese Texte aus ihrem kultischen Rahmen herausgelöst werden und zur "Literatur" einer Gruppe von Eingeweihten werden, die nicht mehr als bevollmächtigte Priester im Auftrag der Gesellschaft, sondern als Individuen in eigener Sache nach Gottesnähe streben. Die Esoterik der Texte korrespondiert dann der Abgrenzung der Gruppe aus dem Ganzen der Gesellschaft. Dieser Schritt wird im Rahmen der altägyptischen Religion vermutlich noch nicht vollzogen, sondern kennzeichnet erst die graeco-ägyptische Phase. Erst jetzt bilden sich Gruppen, die das heilig-magische Wissen nicht im Auftrag der Gesellschaft, sondern in Abgrenzung von ihr, u.U. geradezu gegen sie, verwalten. Damit gewinnt Geheimhaltung einen ganz anderen Sinn.

${ }^{57}$ Man muß hier zwei Dinge unterscheiden: 1. den Akt der Priesterweihe als Initiation ins Priesteramt, und 2. die Kenntnis der heiligen Überlieferungen, die als Eingeweihtsein in streng gehütete Geheimnis dargestellt wird. Ich spreche hier nur von dem zweiten Komplex. 